\title{
Active and passive stabilization of a high-power UV frequency-doubled diode laser
}

\author{
Ulrich Eismann, Martin Enderlein, Konstantinos Simeonidis, \\ Felix Keller, Felix Rohde, Dmitrijs Opalevs, Matthias Scholz, \\ Wilhelm Kaenders, Jürgen Stuhler
}

TOPTICA Photonics AG, Lochhamer Schlag 19, 82166 Graefelfing, Germany

\begin{abstract}
We present a resonantly frequency-doubled tapered amplified semiconductor laser system emitting up to $2.6 \mathrm{~W}$ blue light at $400 \mathrm{~nm}$. The output power is stable on both short and long timescales with $0.12 \%$ RMS relative intensity noise, and less than $0.15 \% / \mathrm{h}$ relative power loss over 16 hours of free running continuous operation. Furthermore, the output power can be actively stabilized, and the alignment of the input beams of the tapered amplifier chip, the frequency doubling cavity and - in case of fiber output - the fiber can be optimized automatically using computer-controlled mirrors.
\end{abstract}

\section{Introduction}

High-power tunable single-frequency lasers around $400 \mathrm{~nm}$ have numerous applications in research and (quantum) technology. These span from laser cooling and trapping of ions (calcium and strontium [1]) or atoms (erbium [2], dysprosium [3], calcium [4] and chromium [5]) to two-photon down conversion or even experiments with antimatter [6]. Furthermore, the 400-nm sources can serve as fundamental lasers for further frequency doubling to the DUV range below $200 \mathrm{~nm}[7]$. While Watt-class output power is required, direct diode lasers are limited to $\sim 100 \mathrm{~mW}[8]$. Frequency doubling of infrared lasers potentially delivers higher power levels, but stability becomes a major concern. Here we present a laser system consisting of a resonantly frequencydoubled tapered amplified semiconductor laser which is capable of emitting 2.6 Watts of single-frequency emission around $400 \mathrm{~nm}$. Based on passive and active measures, we demonstrate stable operation on timescales reaching from hundreds of nanoseconds to many days.

\section{Output power and stability}

The laser system consists of an external cavity diode laser (DL pro), which is amplified with a semiconductor tapered amplifier chip (TA pro) delivering up to $5 \mathrm{~W}$ around $800 \mathrm{~nm}$. The almost diffraction-limited TA pro output beam is mode matched to 
the second harmonic generation (SHG pro) cavity. By choosing an input coupler reflectivity of $98.5 \%$, we optimize the system for SHG output power and we obtain up to 2.6 Watts at $400 \mathrm{~nm}$ with several nanometers of tunability. The cavity is locked to resonance by a digital control loop with a bandwidth of $30 \mathrm{kHz}$ using a slow and a fast piezoelectric transducer (PZT). The necessary error signal is obtained with the Pound-Drever-Hall technique [9] from sidebands created by direct modulation of the DL pro current at $20 \mathrm{MHz}$. Furthermore, the digital control electronics features an automatic relock functionality. The output beam is almost diffraction limited.
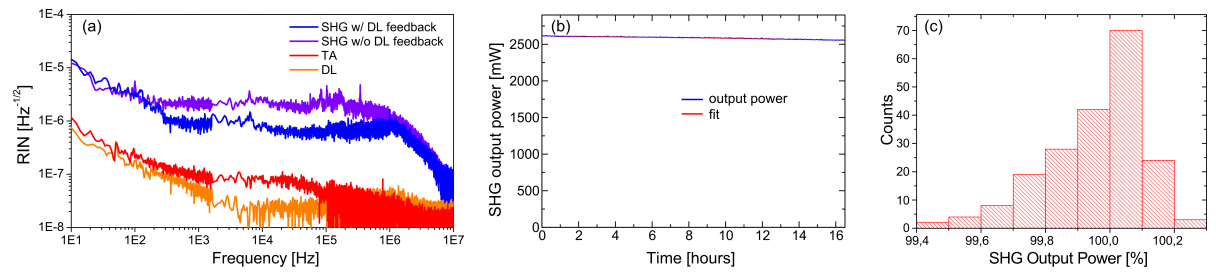

Figure 1: (a) Relative intensity noise (RIN) of different laser output stages. (b) Long-term passive output stability of SHG pro light. (c) Histogram of AutoAlign results. (a)-(c): See text for details.

The results on a relative intensity noise (RIN) measurement of the laser system are shown in Fig. 1 (a). By integrating over the full range $(10 \mathrm{~Hz}$ to $10 \mathrm{MHz})$, we obtain values of $0.01 \%$ RMS for both DL pro and TA pro output by accessing both beams through the respective ports. Due to its finite acceptance range of $f_{\text {acc }} \approx 1 \mathrm{MHz}$, the SHG pro cavity converts frequency noise to RIN. The DL pro has a typical linewidth of $100 \mathrm{kHz}$. Thus, with a 2-stage PZT lock (slow PZT for large displacements, and fast PZT), the RIN of the system at $400 \mathrm{~nm}$ is increased to $0.21 \%$ RMS (SHG w/o DL feedback in Fig. $1(\mathrm{a})$ ), and leveling off at frequencies above $f_{\text {acc }}$, where the cavity acts as a low-pass filter. We improve the RIN value significantly when locking the DL pro to the SHG pro cavity using direct feedback to the laser diode current (SHG w/ DL feedback in Fig. 1 (a)), and obtain a value of $0.12 \%$ RMS.

The SHG pro cavity is of bow-tie type, contains a temperature controlled AR coated non-linear optical crystal and is air-sealed (typ. residual leak rate $<10^{-5} \mathrm{mbar} \mathrm{l} / \mathrm{s}$ ). Together with the advanced mechanical design of the laser system, this yields excellent longterm stability, see Fig. 1 (b). A linear fit, represented by the red line, yields a relative power drop of $0.15 \% / \mathrm{h}$ over more than 16 hours of continuous operation without any adjustments. Furthermore, by applying feedback to the TA pro current, the output power of either TA pro stage, SHG pro stage or the optional fiber can be actively held constant. We observe a beam pointing stability below $2 \mu \mathrm{rad}$ RMS over 60 hours of continuous operation.

Even with the power stability shown in Fig. 1 (b), a time-to-time realignment of the laser system may be necessary in order to achieve optimum output power. Therefore, we have developped an automatic procedure (AutoAlign) relying on a simplex algorithm and a motorization of the patented flexure mirror mounts [10]. The system is optimized stage-by-stage via two-mirror beam walks starting from the coupling of the DL pro beam into the TA pro. In a second step the TA pro beam is aligned to the SHG pro cavity. Finally, in case of fiber output, the coupling into fiber can be optimized. A histogram of the results of 200 AutoAlign procedures is shown in Fig. 1 (c). After deliberate misalignment, the algorithm converges to within fractions of a percent 
of the optimum value. The servos are switched off and completely passive when not in use for the AutoAlign procedure. Thus, no degradation of the laser parameters due to acoustic noise and heat input are observed.

\section{Conclusion and outlook}

We have presented results on a low noise, high power UV laser system allowing handsoff operation for extended periods of time. On the same platform, we have built similar systems with Watt-level power at e.g. $420 \mathrm{~nm}, 486 \mathrm{~nm}, 589 \mathrm{~nm}$ and $671 \mathrm{~nm}$, and up to $2.7 \mathrm{~W} @ 560 \mathrm{~nm}$. Frequency locking of the DL pro master laser allows relative or absolute stabilization to laser references, and offers the potential for Hz-linewidth output [11] for metrology. Furthermore, these sources are efficient fundamental lasers for frequency doubling to the DUV range.

\section{References}

[1] Jürgen Eschner, Giovanna Morigi, Ferdinand Schmidt-Kaler, and Rainer Blatt. Laser cooling of trapped ions. J. Opt. Soc. Am. B, 20(5):1003-1015, May 2003.

[2] JJ McClelland and JL Hanssen. Laser cooling without repumping: a magnetooptical trap for erbium atoms. Physical review letters, 96(14):143005, 2006.

[3] Seo Ho Youn, Mingwu Lu, and Benjamin L Lev. Anisotropic sub-doppler laser cooling in dysprosium magneto-optical traps. Physical Review A, 82(4):043403, 2010 .

[4] Th Kisters, K Zeiske, F Riehle, and J Helmcke. High-resolution spectroscopy with laser-cooled and trapped calcium atoms. Applied Physics B, 59(2):89-98, 1994.

[5] Jürgen Stuhler, Piet O Schmidt, Sven Hensler, Jörg Werner, Jürgen Mlynek, and Tilman Pfau. Continuous loading of a magnetic trap. Physical Review A, 64(3):031405, 2001.

[6] KSE Eikema, J Walz, and TW Hänsch. Continuous wave coherent lyman- $\alpha$ radiation. Physical Review Letters, 83(19):3828, 1999.

[7] Matthias Scholz, Dmitrijs Opalevs, Patrick Leisching, Wilhelm Kaenders, Guiling Wang, Xiaoyang Wang, Rukang Li, and Chuangtian Chen. A bright continuouswave laser source at $193 \mathrm{~nm}$. Applied Physics Letters, 103(5):051114, 2013.

[8] DL pro HP - high power from blue laser diodes, retrieved from: http://www.toptica.com/, 012016.

[9] RWP Drever, John L Hall, FV Kowalski, J Hough, GM Ford, AJ Munley, and $\mathrm{H}$ Ward. Laser phase and frequency stabilization using an optical resonator. Applied Physics B, 31(2):97-105, 1983.

[10] Ulrich Eismann, Matthias Scholz, Tim Paasch-Colberg, and Jürgen Stuhler. Kurz, kürzer, am kürzesten. Physikjournal Best of, page 7, Oktober 2015.

[11] YN Zhao, J Zhang, J Stuhler, G Schuricht, F Lison, ZH Lu, and LJ Wang. Subhertz frequency stabilization of a commercial diode laser. Optics Communications, 283(23):4696-4700, 2010. 